\title{
Intense exercise increases HDL level in children regardless of body weight
}

\author{
Daniel Romero-Gamboa ${ }^{1}$, Victoriano Pérez-Vázquez ${ }^{1}$, Katya Vargas-Ortiz ${ }^{1}$, \\ Francisco J. Díaz-Cisneros ${ }^{2}$, Claudia Martínez-Cordero ${ }^{3}$, Maciste H. Macías-Cervantes ${ }^{{ }^{*}}$
}

${ }^{1}$ Department of Medical Sciences, Health Science Division, University of Guanajuato, León, México;

*Corresponding Author: macistehabacuc@yahoo.com.mx

${ }^{2}$ Apply Work Science Department, Health Science Division, University of Guanajuato, León, México

${ }^{3}$ Hospital Regional de Alta Especialidad del Bajio, León, México

Received 8 October 2013; revised 8 November 2013; accepted 21 November 2013

Copyright (C) 2013 Daniel Romero-Gamboa et al. This is an open access article distributed under the Creative Commons Attribution License, which permits unrestricted use, distribution, and reproduction in any medium, provided the original work is properly cited.

\begin{abstract}
Childhood obesity seems to be increasing at a disturbing rate. Exercise and diet are recommended to combat this epidemic. Light and moderate physical activity is associated with health benefits, whereas intense physical activity is associated with normal BMI and low cardiovascular risk. The aim of this study was to assess the metabolic response to a graded exercise test (GXT) in children with different body weight. Eight boys and nine girls underwent a GXT on a treadmill according to Blake protocol. These children were 7 - 9 years old. The participants were divided into three groups according to their body weight: normal-weight, overweight, and obese. After GXT, lactate and HDL levels increased significantly in all three groups ( $p<$ 0.05). In this experimental trial, we show that HDL level increased after a single session of intense exercise in children regardless of body weight.
\end{abstract}

Keywords: Obesity; Insulin; Physical Activity

\section{INTRODUCTION}

The prevalence of overweight and obesity among Mexican children increased 7\% (from 19\% to $26 \%$ ) in seven years (from 1999 to 2006) according to the National Health and Nutrition Survey [1]. It is well known that the combination of overweight and low physical activity increases levels of glucose, insulin, triglycerides, and LDL. In addition, overweight and low physical activity decrease HDL levels [2]. Exercise and diet have been recommended to combat the childhood obesity epi- demic [3,4]. The Mexican Ministry of Health recommends that children should exercise 60 minutes of moderate aerobic activity more than 3 days per week [3].

There is a growing agreement among experts that increasing physical activity improves health. Moderate and light physical activity has been associated with health benefits, whereas intense physical activity has been associated with lower morbidity and mortality, normal BMI, and low cardiovascular risk [5-7]. Kokkinos (2008) demonstrated that Caucasians and African-Americans with less physical capacity present higher cardiovascular mortality [8]. Intervention programs of twelve-week period of physical activity have shown a decrease in the level of insulin and improvement in lipid profile among overweight and obese children $[9,10]$. Supervised exercise for many weeks has demonstrated being effective to reduce LDL-C and elevate HDL-C levels [11]. On the other hand, obese children presented a negative effect on body composition and lipid profile when they detrained physical activity [12,13]; for example, a day of sitting considerably reduced insulin action in healthy people [14].

Graded exercise testing (GTX) is used to evaluate the responses of different physiological parameters as heart rate, respiratory rate or inspired air during exercise. GTX is a valuable tool to evaluate functional capacity in many settings. In a previous study about the response to a GXT, we reported that overweight children presented a significant decrease in serum insulin level compared with normal-weight children [15]. However, it seems that poor physical fitness is a risk factor for insulin resistance regardless of obesity degree and body composition; a complete physiologic response of children needs to be evaluated. The aim of this study was to assess the metabolic response to a GXT in children with different body weights. 


\section{METHODS}

\subsection{Participants}

Twenty one children ( 7 - 9 years old) started the protocol; they were recruited in an elementary school. Only 17 children (eight boys and nine girls) completed the trial and blood tests. These children were healthy; four children were excluded for musculoskeletal disorders that could affect their performance during a GXT, or for having taken medicine for loss weight. The participants were divided into three groups according to their body weight [16]: normal-weight, overweight, and obese. This study was approved by the ethics committee of Department of Medical Science, University of Guanajuato and all patients and their parents gave their informed written consent. All participants and their parents were informed detailed verbal regarding the purpose of the trial and the study procedures.

\subsection{Measures}

In a previous morning of the test, weight and height of children were measured using a calibrated clinical balance and wall-mounted stadiometer, with a precision of $0.1 \mathrm{~kg}$ and $0.5 \mathrm{~cm}$ respectively. Additionally, a complete clinical history was taken including detailed information on physical activity and diet habits (24-hour food recall). In the test day, participants arrived at Department of Applied Sciences of the University of Guanajuato after an overnight 12-hour fast. A physical examination was done, which included skinfolds and circumferences using the technique described by Lohman. Also, a baseline electrocardiogram was taken to exclude any heart disease than could be contraindicated for a GXT. Systolic and diastolic blood pressures were measured with a proper cuff according arm length. Two blood pressure measurements were taken from participants 10 and 12 minutes before GXT.

\section{3. $\mathrm{VO}_{\text {2peak }}$ Determination}

All children underwent a GXT on a treadmill (Track Master Research). Children were familiarized with exercise procedures and equipment before the beginning of the exercise test. A warm-up period of 3-minute at $0 \%$ grade and $2.8 \mathrm{mph}$ speed was followed of gradual speed increase up to $3.4 \mathrm{mph}$ at 2-minute stages. During the test, the child walked/ran on the treadmill according to Blake protocol (3.4 mph speed $+1 \%$ incline of the treadmill every minute) until the participant was no longer able to continue due the child reached his/her maximal subjective effort (exhaustion) [17]. Throughout the test, heart rate was recorded using a portable heart rate monitor (Polar RS400sd, Polar Electro Inc., Finland). $\mathrm{VO}_{2 \text { peak }}$, the greatest amount of oxygen consumed during strenu- ous aerobic exercise, was calculated for all participants using the formula: $\left(\mathrm{mL}^{*} \mathrm{~kg}^{-1} * \min ^{-1}\right)=11.12+(1.51 *$ exercise time).

\subsection{Biochemical Determinations}

Blood samples were obtained while children were seat. For each child, two venipuncture samples were taken: at baseline and two minutes after ending GXT. Glucose concentration was immediately measured by the glucose oxidase technique, and analyzed at Applied Science laboratory of the University of Guanajuato for total cholesterol, HDL, LDL, triglycerides, hematocrit, and insulin. All the serum lipids were determined using an automatic analyzer (Biosystems) and insulin using radioimmunoassay (Insulin Coat-A-Count, SIEMENS). Additionally, none capillary blood samples were taken to determine blood lactate (Accutrend, Roche Diagnostics, Indianapolis, USA).

\subsection{Statistical Analysis}

All data analyses were performed using Statistics and SPSS software. The data are routinely presented as mean \pm SD. The variables before and after GXT were compared using paired T-test, and the comparisons between groups were analyzed using ANOVA.

\section{RESULTS}

Although total participants were 21 children, only 17 completed the second blood sample ( 9 girls and 8 boys). According to body weight, we classified children in three groups: normal-weight $(\mathrm{n}=6)$, overweight $(\mathrm{n}=5)$, and obese $(n=6)$. Children average age was 7.6 years old. Body weight, triceps, sub-scapular skinfolds and waist circumference were higher in obese group compared to the overweight group, and higher in the overweight group compared to normal-weight group. Systolic and diastolic blood pressures showed a tendency to increase as body weight was higher $\left(\mathrm{F}_{2,14}=7.3, \mathrm{p}<0.01\right.$; Table 1). $\mathrm{VO}_{2 \text { peak }}$ in GXT was $31.7 \pm 4 \mathrm{ml} / \mathrm{kg} / \mathrm{min}$ in normalweight, $29.48 \pm 4.7 \mathrm{ml} / \mathrm{kg} / \mathrm{min}$ in overweight, and $25.2 \pm$ $5.7 \mathrm{ml} / \mathrm{kg} / \mathrm{min}$ in obese (Table 2).

After GXT, lactate and HDL levels increased significantly in all three groups. In the obese group, triglycerides increased significantly (Table 3). Glucose level changed with GXT ( $\mathrm{F}=5.74$ : normal-weight vs. obese was $p=0.04$, overweight vs obese $p=0.02$ ). Mean insulin decreased in the overweight and obese group after GXT; insulin in the normal-weight group did not change (Table 3).

\section{DISCUSSION}

In this experimental trial, we show that HDL level in- 
Table 1. Characteristics of participants according to body weight.

\begin{tabular}{cccc}
\hline & NW & OW & OB \\
\hline N & 6 & 5 & 6 \\
Age (y) & $7.6 \pm 0.8$ & $7.2 \pm 0.4$ & $8 \pm 0.9$ \\
Gender (B/G) & $4 / 2$ & $0 / 5$ & $4 / 2$ \\
Weight (kg) & $26.8 \pm 5.1$ & $32.4 \pm 5.7$ & $43.7 \pm 8.7$ \\
Height (cm) & $128.4 \pm 9.4$ & $129.6 \pm 8.3$ & $135.4 \pm 5.2$ \\
BMI (kg/m²) & $16.1 \pm 1$ & $19.1 \pm 0.9$ & $23.6 \pm 2.9$ \\
Triceps skinfolds (mm) & $9.9 \pm 2.7$ & $16.1 \pm 3.2$ & $22 \pm 4.6$ \\
Subscapular skinfolds (mm) & $6.4 \pm 1.4$ & $12.2 \pm 0.3$ & $19 \pm 9.2$ \\
Waist circumference (cm) & $60.6 \pm 4.4$ & $68.1 \pm 4.9$ & $81.5 \pm 8.7$ \\
Body fat (\%) & $14.7 \pm 3.9$ & $25.3 \pm 2.8$ & $29.9 \pm 4.1$ \\
SBP (mmHg) & $93.5 \pm 5.6$ & $96.4 \pm 4.7$ & $104 \pm 4.1$ \\
DBP (mmHg) & $57.6 \pm 4.3$ & $57.4 \pm 6.5$ & $64 \pm 6.7$ \\
\hline
\end{tabular}

$\mathrm{NW}=$ normal-weight; $\mathrm{OW}=$ overweight, $\mathrm{OB}=$ obese; $\mathrm{B} / \mathrm{G}=$ boy $/$ girl; $\mathrm{BMI}$ $=$ body mass index; $\mathrm{SBP}=$ systolic blood pressure; $\mathrm{DBP}=$ diastolic blood pressure. "Values are the geometric means and standard deviations.

creased after a single session of intense exercise in children, and this increasing was independent of body weight. Although some studies had demonstrated that an increase in HDL is produced as an adaptation to several sessions of exercise, our results showed this increasing since a single workout $[18,19]$.

Because HDL captures free circulating cholesterol and transports it to the liver for excretion, low HDL blood concentration is a cardiovascular risk factor $[18,20]$. In adults, exercise hours correlate positively with HDL
Table 2. Physiological data of the participants according to body weight.

\begin{tabular}{cccc}
\hline & NW & OW & OB \\
\hline $\mathrm{N}$ & 6 & 5 & 6 \\
$\mathrm{HR}_{\text {rest }}(\mathrm{bpm})$ & $73.8 \pm 10.9$ & $81.4 \pm 14.4$ & $79.8 \pm 14.6$ \\
$\mathrm{HR}_{\max }(\mathrm{bpm})$ & $196.8 \pm 18.6$ & $215.4 \pm 6.3$ & $191.8 \pm 16.9$ \\
$\mathrm{Time}(\mathrm{min})$ & $13.8 \pm 2.6$ & $12.2 \pm 3$ & $9.4 \pm 241$ \\
$\mathrm{VO}_{2 \text { peak }}(\mathrm{ml} / \mathrm{kg} / \mathrm{min})$ & $31.7 \pm 4$ & $29.4 \pm 4.7$ & $25.2 \pm 5.7$ \\
\hline
\end{tabular}

$\mathrm{NW}=$ normal-weight; $\mathrm{OW}=$ overweight; $\mathrm{OB}=$ obesity; $\mathrm{HR}_{\text {rest }}=$ resting heart rate; $\mathrm{HR}_{\max }=$ maximum heart rate; $\mathrm{VO}_{2 \text { peak }}=$ maximal oxygen uptake

*Values are the geometric means and standard deviations.

level change; in general, it has been reported that changes in HDL concentration requires at least 10 -week period program. From one to two days after a single exercise session, adults present an elevation in HDL levels while the full effect is an increase in HDL2-c and a decrease in HDL3-c. Therefore, both a single session and an exercise program have independent effects on lipid profile and lipoprotein level in adults. On the other hand, children use a higher proportion of lipids while exercising than adults [21].

There was no significant difference between $\mathrm{VO}_{2 \text { peak }}$ values among the body-weight groups, but only a tendency of low $\mathrm{VO}_{2 \text { peak }}$ in obese children. This tendency could be explained since obese people have less mitochondria in skeletal muscle and a decreased transcription of genes involved in mitochondrial oxidative phosphorylation [22]. $\mathrm{VO}_{2 \max }$ is an inheritable characteristic because, besides environmental factors, maximal oxygen intake has a genetic component. The maximal response to $\mathrm{VO}_{2 \max }$ during exercise is $47 \%$ in children under 9

Table 3. Basal (B) and post-exercise (P) metabolic data of the participants according to body weight.

\begin{tabular}{cccccccc}
\hline & & NW & & OW & & OB & B \\
\hline N & B & P & B & P & B & 6 \\
\hline Glucose (mg/dl) & 6 & 6 & 5 & 5 & $116 \pm 18$ & $104 \pm 7$ & $95 \pm 7$ \\
TC (mg/dl) & $102 \pm 6$ & $107 \pm 8$ & $106 \pm 7$ & $207 \pm 67$ & $145 \pm 21$ & $157 \pm 31$ \\
HDL (mg/dl) & $41 \pm 3^{\mathrm{a}}$ & $50 \pm 6.8$ & $41 \pm 9^{\mathrm{a}}$ & $50 \pm 8$ & $42 \pm 5^{\mathrm{a}}$ & $47 \pm 5$ \\
LDL (mg/dl) & $96 \pm 26$ & $92 \pm 27$ & $148 \pm 78$ & $147 \pm 77$ & $87 \pm 12$ & $87 \pm 22$ \\
TG (mg/dl) & $73 \pm 37$ & $80 \pm 36$ & $100 \pm 54$ & $97 \pm 26$ & $90 \pm 60^{\mathrm{b}}$ & $104 \pm 56$ \\
Lactate (mmol/l) & $1.2 \pm 0.3^{\mathrm{b}}$ & $4 \pm 1.3$ & $2.2 \pm 1.4^{\mathrm{b}}$ & $6.8 \pm 1.9$ & $1.7 \pm 0.5^{\mathrm{b}}$ & $5.2 \pm 1.9$ \\
Htc (\%) & $42 \pm 2.2^{\mathrm{a}}$ & $43.6 \pm 2$ & $40.7 \pm 2$ & $42 \pm 2.4$ & $42.4 \pm 2.6$ & $44.6 \pm 3.2$ \\
Insulin (IU/ml) & $2.2 \pm 3.3$ & $3.0 \pm 2.9$ & $7.5 \pm 2.5^{\mathrm{a}}$ & $3.3 \pm 4.8$ & $11.7 \pm 9^{\mathrm{a}}$ & $8.9 \pm 7.1$ \\
\hline
\end{tabular}

$\mathrm{NW}=$ normal-weight $\mathrm{OW}=$ overweight $\mathrm{OB}=$ obesity; $\mathrm{TC}=$ total cholesterol, $\mathrm{HDL}=$ high density lipoprotein, $\mathrm{LDL}=$ low density lipoprotein, $\mathrm{TG}=$ triglycerides, Htc $=$ hematocrit. ${ }^{\mathrm{a}} \mathrm{P}<0.05$ for differences between basal and post-exercise within body-weight groups. ${ }^{\mathrm{b}} \mathrm{P}<0.01$ for differences between basal and post-exercise within body-weight groups. 
years of age [23].

In addition, acute strenuous exercise may produce dehydration, and therefore an increase in the hematocrit because the reduction of plasma volume. However, the hematocrit did not change significantly in this study, so the observed changes in blood substances concentration was due simply to the acute effect of exercise.

Blood lactate concentration and the perceived exertion are indicators of an intensive anaerobic work, or at least to other similar efforts [24]. Blood lactate is an intermediary in the carbohydrates oxidation that are released into the bloodstream from muscle cells in a quantity that depends of the pyruvate production and removal rate [25]. In our study, the average lactate concentration after GXT was $4.0,6.8$ and $5.2 \mathrm{mmol} / 1$ in normal-weight, overweight, and obese group respectively. Although an established threshold value of blood lactate is $4 \mathrm{mmol} / \mathrm{l}$ after a short maximal exercise, studies have demonstrated that the maximum lactate concentration is lower in children than in adolescents and adults [26,27]. In other words, children can exercise at intensities close to their maximum oxygen intake without accumulating high lactate; $2.5 \mathrm{mmol} / 1$ of blood lactate could correspond to the maximal oxygen consumption in children, as well as the maximal heart rate [28]. The lower rate of lactate production in children compared with adults may due to a lower relative muscle mass, lower level of activity of pyruvate dehydrogenase enzyme, and a lower oxidative phosphorylation rate with its faster elimination [21,29]. All participants in the study succeeded in lactate concentrations corresponding to a maximal exercise.

Blood pressures tended to be higher as the body weight increased; however, all children were within the 50th percentile when it was calculated percentile for age, weight and height. Obesity can raise blood pressures by increasing the vascular tone caused by decreased nitric oxide bioavailability, greater sympathetic tone, and an elevated expression of angiotensinogen by the adipose tissue, which leads to the activation of the renin-angiotensin aldosterone. Children blood pressures usually increase $1-2 \mathrm{mmHg}$ per year. Performing physical activity during an interval of three years may prevent systolic pressure elevation of $1 \mathrm{mmHg}$, as well as a reduction in BMI [5].

Although the sample included children of different body weight, it is worth bearing in mind that the total number of participants was only 17 children. Another possible limitation is that all of them came from a single school. More studies need to be conducted to explore the metabolic response to a GTX in children of different ages and socioeconomic levels.

\section{CONCLUSION}

Metabolic response to a GTX in children increased
HDL level, and this increase was independent of bodyweight. This elevation improves the health of children because it could help in the prevention of the atherosclerotic cardiovascular disease that begins at an early age and progress through the life. Childhood obesity seems to be increasing at a disturbing rate, and this population is vulnerable because children may not be prepared to make health-related choices for their own. This finding should be used to develop a better physical activity program for children knowing that important changes in HDL occur since a first workout.

\section{ACKNOWLEDGEMENTS}

The study was supported by Consejo Nacional de Ciencia y Tecnología del Estado de Guanajuato, Mexico.

\section{REFERENCES}

[1] Oláiz-Fernández, G., Rivera-Dommarco, J., Shamah-Levy, T., Rojas, R., Villalpando-Hernández, S. and HernándezÁvila, M. (2006) Encuesta Nacional de Salud y Nutrición 2006. Cuernavaca, México: Instituto Nacional de Salud Pública.

http://www.insp.mx/images/stories/Produccion/pdf/10072 2 cp3.pdf

[2] Kraja, A.T., Province, M.A., Arnett, D., Wagenknecht, L., Tang, W., Hopkins, P.N., Djousse, L. and Borecki, I.B. (2007) Do inflammation and procoagulation biomarkers contribute to the metabolic syndrome cluster? Nutrition \& Metabolism (Lond), 4, 28.

http://dx.doi.org/10.1186/1743-7075-4-28

[3] Aguilar-Salinas, C.A. and Gomez-Perez, F.J. (2006) The acapulco declaration: A proposal to reduce incidence of diabetes in Mexico. Revista de Investigación Clínica, 58, 71-77.

[4] Hill, J.O., Wyatt, H.R., Reed, G.W. and Peters, J.C. (2003) Obesity and the environment: Where do we go from here? Science, 299, 853-855. http://dx.doi.org/10.1126/science.1079857

[5] Gidding, S.S., et al. (2006) Higher self-reported physical activity is associated with lower systolic blood pressure: The dietary intervention study in childhood (DISC). Pediatrics, 118, 2388-2393. http://dx.doi.org/10.1542/peds.2006-1785

[6] Kimm, S.Y., et al. (2005) Relation between the changes in physical activity and body-mass index during adolescence: A multicentre longitudinal study. Lancet, 366, 301307. http://dx.doi.org/10.1016/S0140-6736(05)66837-7

[7] Ferguson, M.A., et al. (1999) Effects of exercise training and its cessation on components of the insulin resistance syndrome in obese children. International Journal of Obesity and Related Metabolic Disorders, 23, 889-895. http://dx.doi.org/10.1038/sj.ijo.0800968

[8] Kokkinos, P., et al. (2008) Exercise capacity and mortality in black and white men. Circulation, 117, 614-622. http://dx.doi.org/10.1161/CIRCULATIONAHA.107.734764 
[9] Macias-Cervantes, M.H., Malacara, J.M., Garay-Sevilla, M.E. and Diaz-Cisneros, F.J. (2009) Effect of recreational physical activity on insulin levels in Mexican/Hispanic children. European Journal of Pediatrics, 168, 11951202. http://dx.doi.org/10.1007/s00431-008-0907-7

[10] Zorba, E., Cengiz, T. and Karacabey, K. (2011) Exercise training improves body composition, blood lipid profile and serum insulin levels in obese children. Journal of Sports Medicine and Physical Fitness, 51, 664-669.

[11] Hayashino, Y., Jackson, J.L., Fukumori, N., Nakamura, F. and Fukuhara, S. (2012) Effects of supervised exercise on lipid profiles and blood pressure control in people with type 2 diabetes mellitus: a meta-analysis of randomized controlled trials. Diabetes Research and Clinical Practice, 98, 349-360. http://dx.doi.org/10.1016/j.diabres.2012.10.004

[12] Woo, J., Shin, K.O., Yoo, J.H., Park, S. and Kang, S. (2012) The effects of detraining on blood adipokines and antioxidant enzyme in Korean overweight children. European Journal of Pediatrics, 171, 235-243. http://dx.doi.org/10.1007/s00431-011-1518-2

[13] Pangrazi, R.P., Beighle, A., Vehige, T. and Vack, C. (2003) Impact of promoting lifestyle activity for youth (PLAY) on children's physical activity. Journal of School Health, 73, 317-321. http://dx.doi.org/10.1111/j.1746-1561.2003.tb06589.x

[14] Stephens, B.R., Granados, K., Zderic, T.W., Hamilton, M.T. and Braun, B. (2011) Effects of 1 day of inactivity on insulin action in healthy men and women: Interaction with energy intake. Metabolism, 60, 941-949. http://dx.doi.org/10.1016/j.metabol.2010.08.014

[15] Macias-Cervantes, M.H., Diaz-Cisneros, F.J. and Hagang, D. (2007) Acute effect of maximal treadmill exercise upon fasting insuline levels in children with elevated levels of insuline. Medicine \& Science in Sports \& Exercise, 39, S456. http://dx.doi.org/10.1249/01.mss.0000274805.72104.d0

[16] Cole, T.J., Bellizzi, M.C., Flegal, K.M. and Dietz, W.H. (2000) Establishing a standard definition for child overweight and obesity worldwide: international survey. BMJ, 320, 1240-1243. http://dx.doi.org/10.1136/bmj.320.7244.1240

[17] Maud, P. and Foster, C. (2005) Physiological assessment of human fitness. Human Kinetics, Champaign.

[18] Couillard, C., et al. (2001) Effects of endurance exercise training on plasma HDL cholesterol levels depend on levels of triglycerides: Evidence from men of the health, risk factors, exercise training and genetics (HERITAGE) Family Study. Arteriosclerosis, Thrombosis, and Vascular Biology, 21, 1226-1232. http://dx.doi.org/10.1161/hq0701.092137

[19] Elosua, R. (2005) Physical activity. An efficient and un- derused way of preventing cardiovascular disease from childhood to old age. Revista Española de Cardiología, 58, 887-890. http://dx.doi.org/10.1157/13078123

[20] Haney, E.M., Huffman, L.H., Bougatsos, C., Freeman, M., Steiner, R.D. and Nelson, H.D. (2007) Screening and treatment for lipid disorders in children and adolescents: Systematic evidence review for the US preventive services task force. Pediatrics, 120, e189-e214. http://dx.doi.org/10.1542/peds.2006-1801

[21] Riddell, M.C. (2008) The endocrine response and substrate utilization during exercise in children and adolescents. Journal of Applied Physiology (1985), 105, 725733.

[22] Mustelin, L., et al. (2008) Acquired obesity and poor physical fitness impair expression of genes of mitochondrial oxidative phosphorylation in monozygotic twins discordant for obesity. American Journal of PhysiologyEndocrinology and Metabolism, 295, E148-E154. http://dx.doi.org/10.1152/ajpendo.00580.2007

[23] Bouchard, C., et al., (2000) Genomic scan for maximal oxygen uptake and its response to training in the HERITAGE Family Study. Journal of Applied Physiology (1985), 88, 551-559.

[24] Lagally, K.M., et al. (2002) Perceived exertion, electromyography, and blood lactate during acute bouts of resistance exercise. Medicine \& Science in Sports \& Exercise, 34, 552-559. http://dx.doi.org/10.1097/00005768-200203000-00025

[25] Franklin, B.A., Whaley, M.H., Howley, E.T. and Balady, G.J. (2000) ACSM's guidelines for exercise testing and prescription. American College of Sports Medicine, Philadelphia. Lippincott Williams \& Wilkins.

[26] Williams, J.R., Armstrong, N. and Kirby, B.J. (1990) The $4 \mathrm{mM}$ blood lactate level as an index of exercise performance in 11-13 year old children. Journal of Science and Medicine, 8, 139-147. http://dx.doi.org/10.1080/02640419008732140

[27] Denadai, B.S., Greco, C.C. and Teixeira, M. (2000) Blood lactate response and critical speed in swimmers aged 10-12 years of different standards. Journal of Science and Medicine, 18, 779-784. http://dx.doi.org/10.1080/026404100419838

[28] Tolfrey, K. and Armstrong, N. (1995) Child-adult differences in whole blood lactate responses to incremental treadmill exercise. British Journal of Sports Medicine, 29, 196-199. http://dx.doi.org/10.1136/bjsm.29.3.196

[29] Beneke, R., Hutler, M., Jung, M. and Leithauser, R.M. (2005) Modeling the blood lactate kinetics at maximal short-term exercise conditions in children, adolescents, and adults. Journal of Applied Physiology (1985), 99, 499-504. 